\title{
LOS MAPAS CONCEPTUALES \\ DE J.D. NOVAK COMO INSTRUMENTOS PARA LA INVESTIGACIÓN EN DIDÁCTICA DE LAS CIENCIAS EXPERIMENTALES
}

\author{
GONZÁLEZ GARCÍA, F.M.* \\ Departamento de Filosofía y Metodología de las Ciencias. Universidad Pública de Navarra. Campus de \\ Arrosadía, s/n. 31006 Pamplona. \\ * Profesor visitante en la Universidad de Cornell.
}

\section{SUMMARY}

This article is a review of Novak's «concept mapping», used as a tool for didactical research. It emphasizes its efficiency, especially with topics related to teaching/learning processes and planning of both curriculum and instruction. A list of bibliographical references has been carefully selected.

«Concept maps and Vee diagrams analysis can be taught and learned from first grade on to professors with tenure. It is enormously gratifying to me to see senior professors come alive again when they get afresh the idea of making cducative events happen. These people change their minds and their work changes. Here is energy for educative reform».

D. Bob Gowin, 1987.

\section{INTRODUCCIÓN}

En los últimos veinte años, ha habido avances significativos en la comprensión del aprendizaje humano, incluyendo el aprendizaje en la escucla. Se han producido asimismo importantes avances en la comprensión de la naturaleza del conocimiento y de la producción del mismo.

Las investigaciones realizadas en la Universidad de Cornell (EEUU), dirigidas a instituciones escolares, desde la educación primaria hasta el aprendizaje en adultos, han contribuido en gran medjda a estos avances. Fruto de estos programas de investigación ha sido el desarrollo de la primera teoría comprensiva de la educación, descrita en dos libros (Novak 1982, Gowin 1981). Más recientemente, la implementación de la teoria ha conducido al desarrollo de nuevas estrategias para que los profesores (y padres/tutores) ayuden a los alumnos a aprender a aprender (Novak y Gowin 1988).

Estas estrategias de enseñanza/aprendizaje sc llaman «concept mapping» $\mathrm{y}$ «knowledge Vee mapping».

Novak (1985) sostiene que hay un gran potencial de aprendizaje en los seres humanos que permanece sin desarrollar y que muchas prácticas educativas entorpecen más que facilitan la expresión del mismo. El propio Novak (1988) llega a afirmar que el modelo de instrucción y evaluación más frecuente en escuelas y universidades justifica y recompensa el aprendizaje memorístico repetitivo $y$, con frecuencia, penaliza cl aprendizaje significativo.

El conocimiento actual sobre el aprendizaje humano (metaaprendizaje) y los procesos mediante los cuales los seres humanos construyen nuevo conocimiento (metaconocimiento) pueden ayudar a liberar mucho más el potencial intelectual de los alumnos.

En este estudio nos centraremos en la técnica de los mapas conceptuales. Una extensa y rigurosa validación empírica confirma su eficacia como instrumentos para la mejora de los procesos de enseñanza/aprendiraje de las ciencias (Cardemone 1975, Álvarez y Risco 1977, Bogden 1977, Stcwart, Van Kirk y Rowell 1979, Buchweitz 1981, Melby-Robb 1982, Symington y Novak 1982, Laine Gurkey 1982, Minemier 1983, Volmink 1983, Edwards y Fraser 1983, Novak, Gowin y Johansen 1983 , 
Kahle 1984, Robertson 1984, Robertson-Taylor 1985, Kinnear, Gleeson y Comerford 1985, Fraser y Edwards 1985, Fuatai 1986, Ersler 1987, Bar-Lavie 1987, Moreira 1988, Brody, Chipman y Scott 1989, Brody y Koch 1989. Brumsted 1990, Heinze-Fry y Novak 1990, Yaakoby 1990 , Novak 1991).

\section{BASES PSICOLÓGICAS PARA EL META. APRENDIZAJE}

David Ausubel, en su Psychology of Meaningful Verbal Learning (1963) y más tarde Educational Psychology: A Cognitive View (1968), presenta una comprensiva y coherente teoría cognitiva del aprendizaje que está explícitamente dirigida hacia el aprendizaje humano, especialmente en instituciones escolares. Posteriormente, tras una década de investigación, fue parcialmente modificada (Ausubel, Novak y Hanesian 1978).

La idea clave de la teoría de Ausubel es la naturaleza del aprendizaje significativo en contraste con el aprendizajc memorístico.

Según Ausubel el aprendizaje significativo requiere:

1. Materiales de aprendizaje significativos.

2. Una disposición por parte de la persona que aprende a enlazar cada concepto del nuevo material con conceptos que ya tiene.

3. Una estructura cognitiva relevante o apropiada en el alumno, es decir, que algunos conceptos de la misna puedan ser relacionados, de manera no arbitraria, con los nuevos conceptos.

La cumplimentación de estas condiciones, desde un puntode vista didáctico/metodológico, parece fundamental, pues implica, de una parte, el conocimiento de la estructura cognitivadel alumno, de otra, la planificación adecuada del currículo y la instrucción y, finalmente, el fomento de actitudes favorables a cste tipo de aprendizaje, con el potencial de motivación necesario.

Fl principio del aprendizaje significativo incluye la idea de que cada uno de nosotros tiene una secuencia única de experiencias de aprendizaje y por consiguiente adquiere significados idiosincrásicos para los conceptos.

Nusubel utilizó la palabra inclusor o concepto inclusor para designar la unidad funcional en la memoria de cada persona.

En algunos casos, este significado idiosincrásico se aparta del significado aceptado culturalmente y decimos que la persona tiene un error conceptual o estructura alternativa. Una vez establecidos en la estructura cognitiva, estos inclusores no son fácilmente modificados (Ficlm y Novak 1983). Otras tres ideas básicas forman el núcleo de la teoría del aprendizaje de Ausubel y juntas sirven para explicar la mayoría de los fenómenos cognitivos.
A medida que el nuevo conocimiento es adquirido mediante aprendizaje significativo, los conceptos inclusores experimentan diferenciación progresiva.

Otra idea clave es que cuando los significados de dos o más conceptos aparecen relacionados de una manera nueva y sifnificativa tiene lugar una reconciliación integradora.

Finalmente el aprendizaje supraordenado se produce cuando se adquiere un nucvo significado conceptual que sirve para integrar el significado de dos o más conceptos.

\section{LOS MAPAS CONCEPTUALES Y EL APREN. DIZAJE SIGNIFICATIVO}

En la mayor parte de los programas escolares, es frecuente que los alumnos memoricen mecánicamente definicionesonomas de procedimiento sin relacionar los significados de las palabras de las definiciones o de las normas con las ideas que ellos ya comprenden.

En realidad, los alumnos llegan a creer que la memorización de la información escolar es la única forma de aprender. Como profesores podemos querer disminuir el aprendizaje memoristico, pero, frecuentemente, nos encontramos impotentes para lograrlo e incrementar el aprendizaje significativo.

Dos importantes razones justifican la dificultad del problema:

1. El alumno no es consciente de que hay una alternativa al aprendizaje memorístico por repetición mecánica.

2. Los conceptos que van a aprenderse son presentados de forma que favorecen la memorización.

Novak y otros (1980) diseñaron el programa Aprendiendo a Aprender, basado en la teoría cognitiva del aprendizaje de Ausubel (1978), para su utilización simultánea junto con los programas regulares de instrucción. Aunque los materiales del programa fueron preparados para los niveles de ciencias de escuela secundaria, han sido implementados con éxito, también, en otros niveles y materias.

El objetivo principal del programa fuc conseguir que los alumnos aprendiesen significativamente.

Para que esto realmente se logre es necesario que los alumnos incrementen su conocimiento de:

1. El proceso de aprendizaje.

2. La naturaleza del conocimiento.

3. Cómo extraer significados de los materiales estudiados.

Los mapas conceptuales están basados en la teoría del aprendizaje de Ausubel-Novak (1978), y fueron diseñados por Novak en 1975. 
Desde entonces, numerosas investigaciones han puesto de manifiesto que la elaboración de mapas conceptuales ayuda a lograr un aprendizaje significativo.

Pensamos con conceptos. Los significados de nuestros conceptos de hechos u objetos cambian con el tiempo, puesto que aprendemos acerca de una variedad más amplia de ejemplos y relacionamos unos conceptos con otros de nuevas maneras.

Existen palabras como: son, donde, el, es, entonces, con, etc., que no corresponden a conceptos, se denominan "palabras de enlace». Son utilizadas junto con los conceptos para construir frases que tienen significado: «proposiciones».

El conocimiento que tenemos de un área determinada consiste en la construcción de conceptos de aquel área en un sistema coherente y ordenado (Novak 1980). Este sistenta puede ser simbolizado mediante la claboración de mapas conceptuales.

Un mapa conceptual puede ser considerado como una representación visual de la jerarquía y las relaciones entre conceptos contenidas en la mente. Para Stewart y otros (1979), el mapa conceptual es un instrumento para representar la estructura conceptual de una disciplina o segmento de una disciplina, en dos dimensiones. Esta circunstancia hace que sea muy apropiado pasa mostrar las relaciones proposicionales entre conceptos. El mapa conceptual facilita el intercambio necesario entre el profesor y el alumno, revelando qué conceptos cstán presentes en el material de enseñanza y en el alumno.

El aprendizaje es compartición de significados (Gowin 1981) y los mapas conceptuales revelan esos significados.

Los mapas conceptuales serían análogos en cierto modo a los mapas de carreteras. Los conceptos representarían las ciudades y las proposiciones las carreteras que las cnlazan. Además no todas las ciudades tienen la misma densidad de población, ni los conceptos del mapa, idéntico poder explicativo o de generalidad.

La representación de las relaciones entre los conceptos en el mapa conceptual, sigue un modelo que va «de lo general a lo específico». Cada mapa conceptual debería tener los conceptos más generales o inclusivos en la parte superior, y los conceptos más específicos en la inferior.

Otro rasgo característico del mapa conceptual es la jerarquía conceptual, que se produce cuando dos o más conceptos se ilustran bajo uno más inclusivo.

Novak y Gowin (1988) en su libro Aprendiendo a Aprender proporcionan abundante y precisa información acerca de la claboración de los mapas conceptuales, así como de todo tipo de sugerencias prácticas y ejemplos.

\section{LOS MAPAS CONCEP'TUALES Y IAA INVES- TIGACION EN DIDACTICA DE LAS CIEN- CIAS}

Relaciono en este apartado una selección de investiga ciones educativas en las que se ha utilizado la técnica de los mapas conceptuales. Sin ánimo de ser exhaustivos, constituye, en nuestra opinión una muestra suficientemente significativa de la eficacia de su utilizacion en investigaciones relacionadas, entre otras, con el currículo, la instrucción y la evaluación.

Siguiendo un orden cronológico citaremos, en primer lugar a Cardemone (1975), quien, investigando con alımnos de College de primer año en un curso de matemáticas, encontró que aquéllos que utilizaron la técnica instruccional de los mapas conceptuales, no sólo obtuvieron mejores resultados en los tests de solución de problemas sino que, además, aumentaron la confíanza en su habilidad para las matemáticas.

Álvarez y Risco (1977) utilizaron con gran exito la técnica de los mapas conceptuales con niños pertenecientes al grado de enseñanza primaria.

Bogden (1977) halló que los mapas conceptuales preparados por el instructor de Genética cn College eran de pequeño valor, pero aquellos alumnos que intentaron utilizar los mapas conceptuales e introdujeron algunas modificaciones propias los encontraron útiles. Se comprobó asimismo la eficacia de los mapas en la planificación de preguntas de examen. Los mapas preparados por Bogden no mostraban palabras de enlace sobre las líneas que unen los conceptos, y csto provocó confusión en los alumnos.

El estudio puso en evidencia que los alumnos deben construir sus propios mapas conceptuales, y tener cuidado y ser explícitos al rotular las lineas cntre conceptos. Asimismo, se constató la importancia de elaborar mapas con una buena «jerarquía conceptual» y evjtar la tendencia a construir "diagramas de flujo», que presentan secuencias de acontecimientos en vez de relaciones supraordenadas-subordinadas entre conceptos.

Stewart, Van Kirk y Roweli (1979) enfatizan el valor (ie la técnica de los mapas conceptuales como un recurso para el diseño de material curricular, planificación instruccional o la evaluación en el campo de la Didáctica de Ia Biología; todo ello justificado por una validación empírica importante.

Para estos autores, los mapas conceptuales representan un enlace entre la teoría del aprendizaje y la enseñanza.

Basados en la teoría de Ausubel-Novak (1978), la eficacia de los mapas conceptualcs reside en que aquélla tiene como objetivo específico el aprendizaje que se produce en instituciones escolares.

Buchweitz (1981) desarrolló mapas conceptuales para cada uno de los seis experimentos de laboratorio utilizados en un curso universitario de Física, sobre electrici* 
dad y magnetismo. Encontró que dos đe los seis experimentos tenían serias deficiencias, en términos de una falta de nuevos enlaces conceptuales desarrollados a través de los acontecimientos observados, o imporłantes omisiones en el conocimiento conceptual/teórico requerió por los alumnos para comprender estos acontecimientos. Utilizando datos de 403 alumnos inscritos, Buchweitz constató que no tuvo Iugar una mejora significativa đel rendimiento en Física en las dos sesiones de laboratorio con serias deficiencias, pero se pusieron de manifiesto progresos altamente significativos en las cuatro sesiones de laboratorio que fueron conceptualmente y metodológicamente interpretables por los alumnos. Sus datos fueron utilizados para revisar el programa de Iaboratorio de los años siguientes.

A alumnos retrasados a menudo se les niega la oportunidad de realizar prácticas de laboratorio, ya que tienden a estar «perdidos» y a menudo se comportan mal. En los trabajos de Melby-Robb (1982) y del grupo de la Lniversidad de Purdue (Kahle 1984), se demuestra que los alumnos con aprendizaje lento y otros pertenecientes a minorías, se centran en las tareas del trabajo de laboratorio y son efectivos, cuando se utilizan instrumentos metacognitivos como los mapas conceptuales.

Symington y Novak (1982) opinan que los mapas conceptuales ayudan a los profesores y a los altumnos a ver la naturaleza conceptual y proposicional del conocimiento y su relación con la comprensión humana. Incluso los niños aprenden fácilmente a construir mapas conceptuales de lecturas de textos, listas de palabras de conceptcs importantes, discusiones en clase sobre experimentos, viajes al campo o de cualquiera otra fuente de experiencias. Además, los mapas conceptuales ayudan a los alumnos a «aprender a aprender».

Gurley (1982), trabajando con alumnos de Biología de una escucla secundaria que utilizaban mapas conceptuales. encontró que entre el 90 y $95 \%$ de los mismos se centraban y ponfan interés en el trabajo de laboratorio, mientras que aquellos porcentajes bajaban a un $40-45 \%$ en alumnos que no usaban dicha estrategia. Además, Ios alumnos declararon ser muy conscientes de su responsabilidad para aprender y del valor a largo plazo del aprendizaje significativo sobre el realizado por repetición mecánica, que era su mođelo ordinario.

Minemier (1983) llegó en sus investigaciones a las mismas conclusiones que Cardemone en 1975. La instrucción con mapas conceptuales llevó al alumno a mejorar sus puntuaciones y a aumentar su confianza en sus propias posibilidades para el aprendizaje de las Matemáticas.

Para Volmink (1983), Ias Matemáticas constituyen una de las materias más «conceptualmente opacas» enseñadas en las escuelas $y$, probablemente, debido a ello muchos alumnos encuentran las matemáticas desagradables y no recuerdan casi nada práctico y útil unos meses después del curso. La utilización de los mapas conceptuales provocó en los alumnos una respuesta positiva, debido a la comprensión conceptual que aquélla lleva asociada.
Edwards y Fraser (1983) consideran que los mapas conceptuales suponen una técnica para revelar la estructura cognitiva que parece manejable dentro de las limitaciones actuales de la clase. Estos autores realizaron un estudio piloto que investigó la viabilidad de la utilización de los mapas conceptuales en las clases de ciencias de la escuela secundaria. Las conclusiones demostraron que los mapas conceptuales tienen un gran potencial como método en clase para revelar la comprensión conceptual de los alumnos, siendo para este propósito tan seguro como las entrevistas. Además se señala la influencia positiva de los mapas conceptuales sobre el aprendizaje y las actitudes de los alumnos.

Novak, Gowin y Johansen (1983), en investigaciones realizadas con alumnos de escuela secundaria, entraron que éstos (de cualquier nivel de habilidad medido por Ios tests estandarizados SAT o SCAT) podían tener éxito en la instrucción mediante mapas conceptuales. La realización de entrevistas clínicas a los alumnos permitió constatar que éstos consideran que las estrategias de aprendizaje que requieren comprensión de la materia constituyen un trabajo duro, pero la mayoría de los estudiantes entrevistados reconocen y valoran la comprensión por encima del aprendizaje memorístico por repetición mecánica. Entre las conclusiones del estudio, Ios autores señalan que los alumnos pueden aprender a utilizar los mapas conceptuales junto con los programas existentes en ciencias.

Los datos obtenidos sugieren además que los alumnos que mejor comprenden los temas científicos que están estudiando no son necesariamente los que consiguen las mejores puntuaciones en los tests estandarizados o en los exámenes típicos de clase, y viceversa. La instrucción con mapas conceptuales fue eficaz también en la mejora de la habilidad de los alumnos para resolver problemas.

Robertson (1984) encontró en sus investigaciones que la mayoría de alumnos mantenían actitudes negativas hacia el trabajo de laboratorio y no comprendían los propósitos del mismo. Sin embargo, cuando fueron utilizados mapas conceptuales con la misma población de alumnos en un estudio posterior, se detectaron actitudes altamente positivas y elevados niveles de comprensión (RobertsonTaylor 1985).

Kinnear, Gleeson y Comerford (1985), teniendo en cuenta el avance experimentado por el uso de hardware $y$ software como recursos para la enseñanza de las ciencias, y que la utilización de actividades basadas en el ordenador estaba moviéndose desde consideraciones logísticas - como acceso a hardware - a otras educativas -como la evaluación de la contribución de actividades basadas en el ordenador al desarrollo conceptual de los alumnos-, utilizaron los mapas conceptuales para medir el valor de una actividad basada en el ordenador con la intención de favorecer la comprensión conceptual de los alumnos y en particular decidir cómo esta actividad debería integrarse con otras componentes de una sesión de laboratorio.

El análisis de los mapas conceptuales reveló un incremento en la comprensión conceptual de los alumnos que realizaron la actividad basada en el ordenador. 
Los autores recomiendan la introducción de este tipo de actividades en las experiencias de aprendizaje de los alumnos, puesto que facilitan la comprensión de los conceptos.

Fraser y Edwards (1985) diseñaron una investigación para probar si el perfeccionamiento en el uso de la técnica de los mapas conceptuales modificaría el rendimiento del alumno en los exámenes tradicionales de clase.

De las conclusiones se desprende la existencia de una correlación positiva entre la realización de los mapas por parte del alumno y una mejora en los resultacios de los exámenes.

Además, se comprueba en el estudio que los alumnos que habían conseguido un alto nivel en el dominio de la técnica experimentaron progresos significativos.

Ftratai (1986) encontró en sus investigaciones que alumnos de escuela secundaria, en la Samoa americana, después de la instrucción con mapas conceptuales, no sólo obtuvicron mejores puntuaciones en los tests de Matemáticas típicos, sino que también aumentó su habilicad para la resolución de problemas matemáticos nuevos.

Ersler (1987) utilizó mapas conceptuales en tres fases de su investigación: diseño del currículo, enseñanza y evaluación. Mediante el uso de los mapas, analizó la estructura conceptual de la materia para ser tenida en cuenta en las lecciones.

Los mapas conceptuales realizados por los alumnos fueron empleados para identificar el conocimiento de los mismos antes de la instrucción, para guiar el desarrollo de las lecciones y como medio para evaluar cl cambio conceptual que sigue a la instrucción.

Los mapas conceptuales, dibujados por el autor de la investigación en la pizarra, se utilizaron también como recurso instruccional en algunas de las lecciones de clase.

También Firsler, trabajando con alumnos de $5^{2}$ grado, encontró que prácticas de laboratorio y experiencias de campo dieron lugar a cambios actitudinales muy positivos hacia la valoración de un pantano local, y a significativos incrementos en la comprensión de conceptos ecológicos, especialmente en alumnos con un mejor conocimiento inicial, y todo ello cuando se utilizaban mapas conceptuales.

Bar-Lavie (1987) obtuvo en Israel resultados muy positivos con alumnos de grado 11 (16-18 años), utilizando la técrica de los mapas conceptuales. La implementación de las unidades instruccionales diseñadas pusieron en evidencia las diferencias entre el aprendizaje significativo y el aprendizaje memorístico por repetición mecánica, y la necesidad del alumno de responsabilizarse de su propio aprendizaje.

Bar-Lavie observó también, que los alumnos que aprendieron significativamente fueron más positivos en sus actitudes hacia el estudio.
Moreira (1988) ha realizado numerosas investigaciones siguiendo el marco referencial de la Teoría de la Educación de Novak, especialmente en el campo de la Didáctica de la Física. Él expone en sus publicaciones puntos de vista y puntualizaciones de tipo práctico muy acertadas. En nuestra opinión, es un autor muy interesante y, por tanto, le vamos a dedicar una atención especial.

Moreira propone Ios mapas conceptuales como instrumentos potencialmente útiles para la enseñanza, evaluación y estructuración de los planes de estudio. Define los mapas conceptuales como: «Diagramas bidimensionales que muestran relaciones jerárquicas entre conceptos de una disciplina y que derivan su existencia de la propia estructura de la disciplina». Un mapa conceptual debe verse como «un mapa conceptual», y no como cel mapa conceptual» de un determinado conjunto de conceptos. Cualquier mapa conceptual debe ser considerado como una de las posibles representaciones de una cierta estructura conceptual.

Para Moreira no hay un modelo único de mapa conceptual y no existen reglas fijas que deban ser observadas al construir un mapa. Considera que el criterio «de Io general a lo específico" en la construcción de mapas, especialmente cuando cl número de conceptos es elevado y el mapa necesariamente amplio, es difícil de cumplir; y lo mismo ocurre en el caso de evaluaciones finales o sesiones integradoras de conceptos, después de un período de instrucción más o menos largo, correspondiente al desarrollo de unidades didácticas del currículo.

Moreira (1988) resume las aplicaciones de los mapas conceptuales en tres ámbitos, como recursos de enseñanza, como instrumentos de evaluación y como auxiliares en la planificación de los programas de estudio. Como recursos de enseñanza, los mapas conceptuales presentan las relaciones jerárquicas entre los conceptos que son enseñados en clase, facilitando el aprendizaje de los mismos. Desde el punto de vista educativo, la utilización de los mapas no debe ser exclusivamente de arriba a abajo, ya que la enseñanza debe ser planeada además para promover «reconciliación integradora». Según Novak (1982), ésta se consigue de una manera más eficaz, cuando la enseñanza se organiza de tal forma que «baje y suba» en las jerarquías conceptuales a medida que se presenta nueva información.

En otras palabras, se debe «bajar y subir» en el mapa, explorando explícitamente las relaciones de subordinación y supraordenación entre los conceptos (Moreira y Masini 1982).

Moreira cita también, algunas desventajas de los mapas conceptuales que el profesional docente debe tratar de subsanar. Entre éstas se encuentran:

- que el mapa no tenga significado para los alumnos; - que los mapas sean excesivamente confusos, con muchas líneas.

Los mapas conceptuales hechos por el profesor pueden inhibir la habilidad de los alumnos para construir sus propias jerarquías conceptuales. 
El propio Moreira sugiere soluciones como:

- Introducir los mapas cuando el alumno esté familiarizado con el tema.

- No trazar todas las posibles líneas que indiquen relaciones entre conceptos, en aras a una mayor claridad.

La utilización de los mapas conceptuales como instrumentos de evaluación đevuelve a la misma su verdadero sentido, el de obtener información sobre el tipo de estructura que el alumno ve para un conjunto de concep- tos dado. Para ello se le puede pedir que construya el mapa o bien éste puede construirse indirectamente a partir de entrevistas o de sus respuestas a exámenes escritos (Moreira y Novak 1988).

Si tenemos en cuenta que el conocimiento previo del alumno es el factor que más influye en el aprendizaje posterior (Ausubel 1978) es obvio que la determinación de esos conocimientos previos es importante, y para ese fin, los mapas conceptuales son muy útiles para el profesor, pues reflejan, en buena medida, lo que el alumno ya sabe (Figs. 1 y 2 ).

Figura 1

Mapa conceptual realizado a partir de conceptos relevantes seleccionados de los contenidos correspondientes a la unidad didáctica $\mathrm{n}^{\mathrm{n}} 5$ : Los silicatos, del programa de Geología del curso $2^{\circ}$ de Ciencias de la Escuela Universitaria de Formación del Profesorado de EGB de la Universidad Pública de Navarra. Se ha utiłizado el Programa CMap creado por Hunter, S. y Stahl, H. (1986), para ordenador Macintosh.

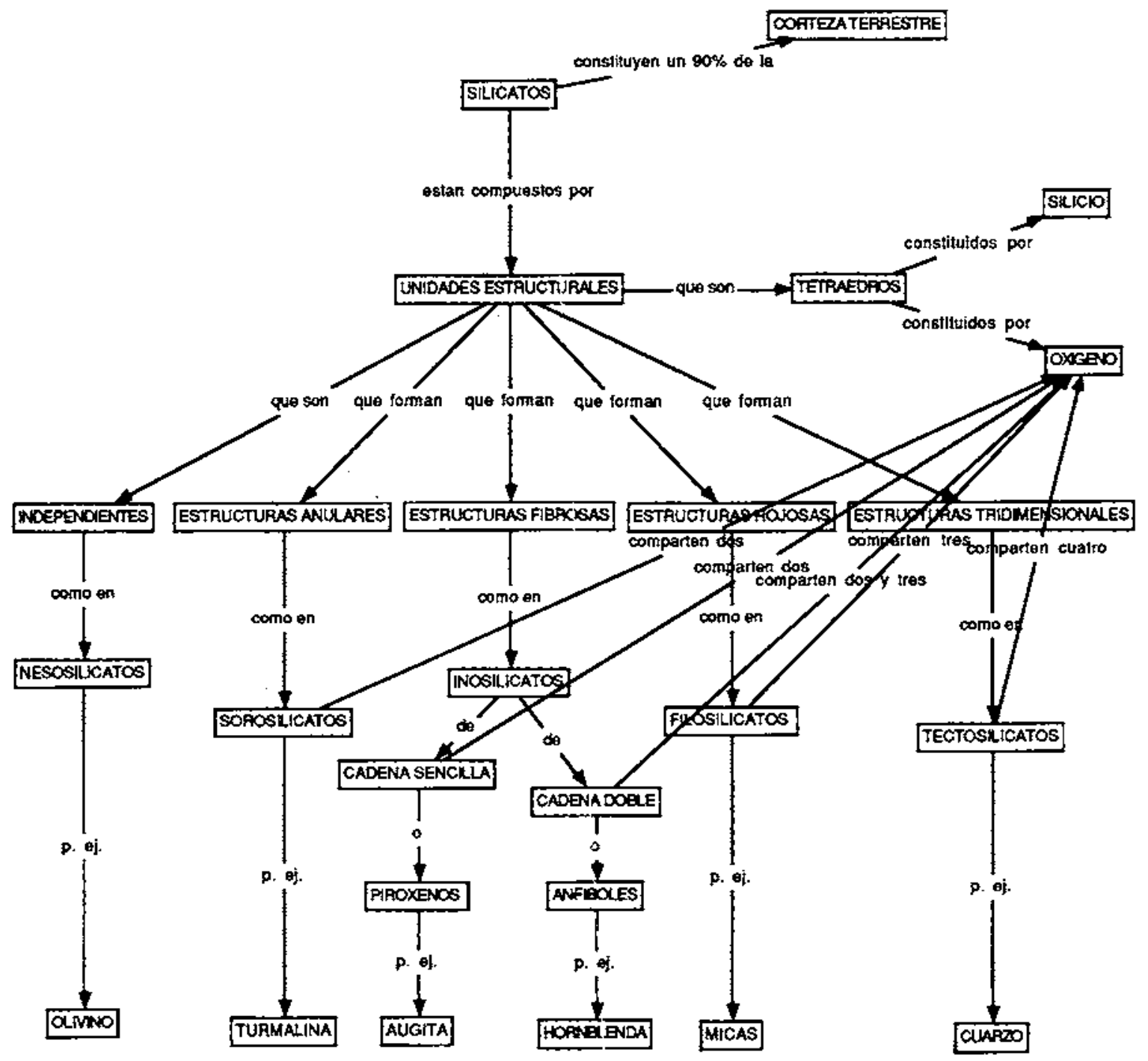


Figura 2

Mapas conceptualts realizados por alumnos de Ceologia del curso $2^{2}$ de ciencias de la Fiscuela Liniversitaria de Formación del Profesorado de EGB de Pamplona, utilizando los mismos conceptos que en la Figura l. La elaboración de los mapas fue anterior al desarrolio de los procesos de instrucción correspondientes y se llevó a cabo sin información adicional alguna concerniente a los temas respectivos. (bssérvese la eficacia de los mapas conceptuales para revelar los conocimientos previos del alumno, requisito indispensable para cl profesor en orden a inducir un aprendizaje significativo en sus alumnos.

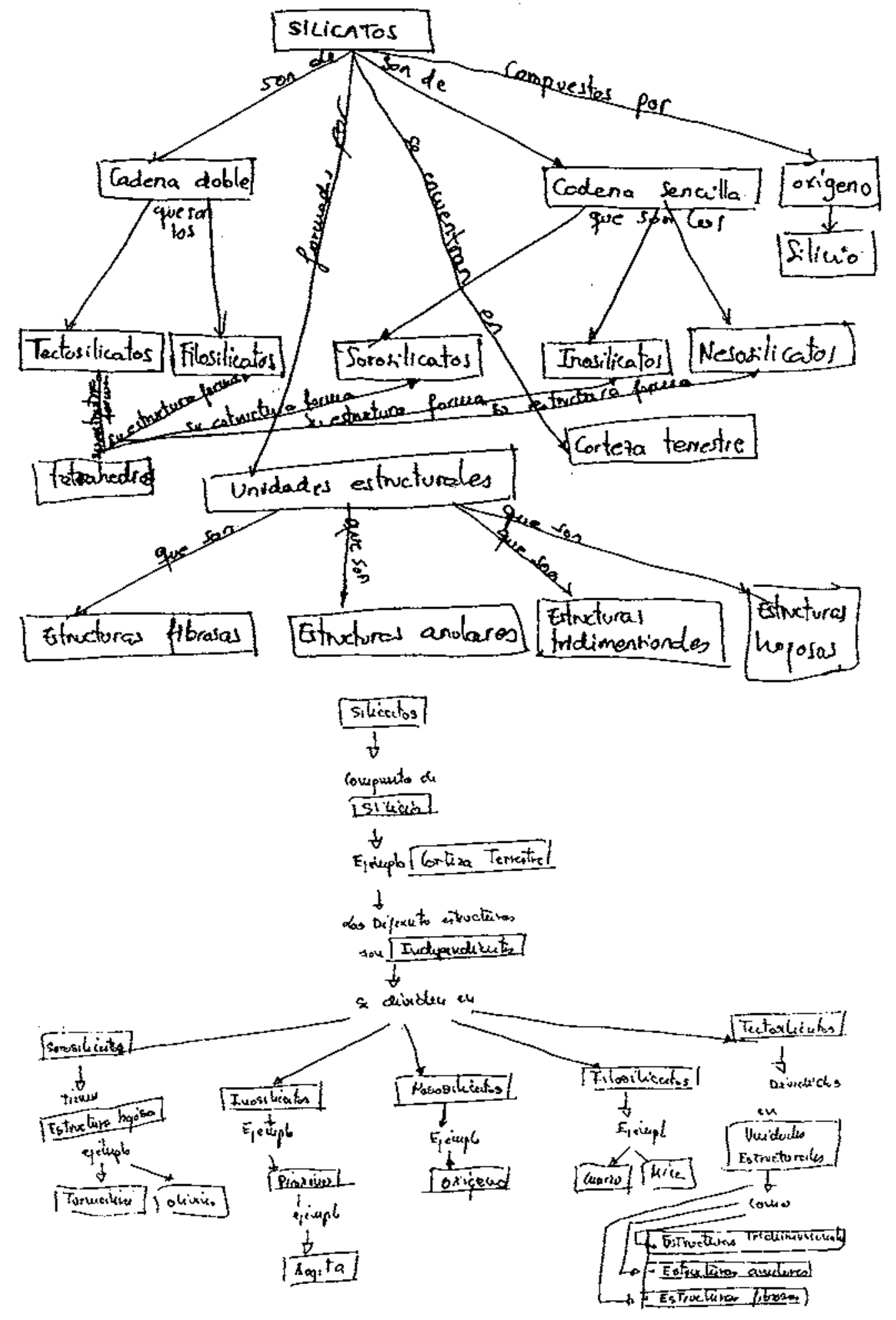


El hecho de que el mapa conceptual sea una representa ción de la estructura cognoscitiva del alumno nos va a permitir su utilización para medir los cambios en la misma a medida que se realiza la enseñanza.

I a información que proporcionan los mapas conceptuales puede y dcbe ser terida en cuenta en el rediseño de estrategias de instrucción y de contenidos curriculares, de forma que se favorezca una correcta construcción de conocimientos por parte del alumno. Los mapas conceptuales pueden servir como recursos en la planificación de los programas de estudio.

Dependiendo del grado de generalidad o especificidad de los conceptos, de su nivel de inclusividad, los mapas conceptuales pueden ser útiles para la programación desde una clase hasta toda una carrera.

Para Stewart y otros (1979), los mapas conceptuales son importantes para centrar la atención dentro del progra$\mathrm{ma}$, en la distinción entre el contenido que se cspera que sca aprendido y aquél-instrumental-que servirá de vehículo para cl aprendizaje.

Una buena programación requiere un análisis đe cuáles son los conceptos más importantes para la comprensión de la disciplina. Los mapas pueden ayudar enormemente en esta labor, así como a evitar solapamientos y repeticiones inútiles de conceptos, cn la misma materia y en materias diferentes.

Finalmente, otro de los aspectos importantes, desde el punto de vista cducativo, lo constituye el hecho de que los mapas conceptuales son instrumentos titiles para la negociación de significados (Novak y Gowin 1988), puesto que son una representación explícita, abierta, de los conceptos y proposiciones que tiene una persona; los mapas conceptuales permiten que los alumnos y los profesores intercambien, «negocien» los significados hasta que los compartan (Gowin 1981).

Brody, Chipman y Scott (1989) utilizaron mapas conceptuales para la selección y organización de los contenidos en rclación con un tema de gran actualidad en educación ambiental: la Iluvia ácida.

Además sirvieron para cl diseño de materiales curriculares de educación ambiental, basados en el conocimiento, del alumno, una vez detectado mediante cntrevistas errores conceptuales y carencias en el conocimiento de determinados conceptos.

Brody y Koch (1989) emplearon los mapas conceptuales, en esa misma línea, para organizar la información acerca de ciertos temas y como material posterior de discusión y análisis, en orden a la selección de los contenidos y a la obtención de los principios considerados esenciales para la comprensión de los temas.

Brumsteđ (1990) realiza un análisis descriptivo sobre cómo los métodos de enseñanza y la sclección de la materia afectan al compromiso del alumno de enseñanzá secundaria en clase. Los mapas conceptuales fueron utilizados para reunir información y para evaluar el aprendizaje de los alumnos y los cambios de actitud.

Uno de los objetivos que merece la pena en educación es alejar a los alumnos del aprendizaje memorístico, por repetición mecánica, y acercarlos al aprendizaje significativo. Heinze-Fry y Novak (1990) investigaron Ia utilización de los mapas conceptuales como un instrumento para facilitar el aprendizaje significativo. Además el estudio midió las actitudes de los alumnos hacia los mapas conceptuales. Parece claro, en las condiciones de la investigación, que cl aprendizaje más activo por parte de los alumnos que la realización de los mapas conceptuales propicia, faculta a aquéllos a moverse hacia un aprendizaje más significativo.

Yaakobi (1990) presentó en Israel una alternativa a aspectos del currículo y de planificación de la instrucción del sistema educativo en Isracl, proponiendo un enfoque de organización psicológica en vez del enfoque de organización lógica utilizado por los redactores. Yaakobi justificó su acción en el hecho de que a partir de los datos de la litcratura de investigación se deduce la tendencia đe los estudiantes a no integrar los temas que se enscñan separadamente.

Este autor sostiene que sus decisiones sobre el currículo estuvieron influenciadas por los mapas conceptuales.

El uso de los mapas le capacitó para construir los centros de aprendizaje con una organización psicológica. El propósito de la construcción de dichos centros fue doble:

1) Demostrar los pasos implicados en el desarrollo de materiales de aprendizaje relacionados con las teorías de Ausubel, Novak y Gowin, sobre enseñanza y aprendizaje.

2) Animar a los profesores a utilizar materiales de aprendizaje que tengan el potencial necesario para facilitar el aprendizaje significativo.

Desde 1975, Novak y sus equipos de investigación han empleado la técnica de los mapas conceptuales en la mayorfa de sus trabajos, principalmente como un instrumento para analizar y representar la estructura proposicional y conceptual de una materia de estudio o para representar una parte de la organización cognitiva de un alumno determinado.

Fin algunos de sus trabajos los mapas conceptuales se construyeron a partir de las proposiciones manifestadas por alumnos durante una entrevista clínica, mientras que otros estudios utilizaron mapas conceptuales construidos por alumnos, después de ser instruidos en la técnica.

Novak ha idcado asimismo varias claves de puntuación numérica para la evaluación de los mapas conceptuales, facilitándose así un ulterior tratamiento estadístico (Novak y Gowin 1988).

Se ha constatado además que la instrucción con mapas conceptuales ha tenido éxito con alumnos desde seis años de edad hasta adultos. 


\section{A MODO DE CONCLUSIÓN}

Las aplicaciones de los mapas conceptuales de Novak a la mejora de la investigación en la Didáctica de las Ciencias (ver número especial del Joumal of Research in Science Teaching de diciembre de 1990 dedicado monográficamente a «Perspectives on Concept Mapping»), y el desarrollo del heuristico, ba llevado a la NARST a otorgar excepcionalmentc al profesor Novak, como reconocimiento a sus méritos el "Distinguished Contributions Award».

Tomando opción claramente por el modelo constructivista y teniendo en cuenta el énfasis en cl aprendizaje significativo detectado en los fundamentos psicopedagógicos de la Reforma Educativa en España, así como las implicaciones que este tipo de aprendizaje tiene, nuestra experiencia en investigación en Didáctica de Ciencias (González 1991) ha puesto de manifiesto la familiarización rápida de los alumnos con esta técnica instruccional, y la utilidad de la misma en la detección de Ios conocimientos previos del alumno, en el subsiguiente diseño de material curricular e instruccional conceptualmente más claro, así como en la génesis de actitudes positivas generalizadas en los alumnos, los cuales se sienten facultados para «aprender a aprender significativamente», y respetados y valorados en su trabajo.

En cuanto a implicaciones de la utilidad de los mapas conceptuales para el futuro de la investigación en Didáctica de las Ciencias, considero que sería conveniente ahondar en los aspectos anteriores, evaluando en la realidad de nuestro contex to escolar la consistencia del heurístico. Como dice Novak (1991), queda mucho ca* mino por recorrer para determinar la fuerza de los mapas conceptuales para hacer la ciencia conceptualmente transparente a los profesores y, a su vez, a sus alumnos.

\section{REFERENCIAS BIBLIOGRÁFICAS}

KI.VAREL, M. y RISCO, V., 1987. Concept Maps and Vee Diagrams: A Vistal Kepresentation of Children's Thinking. Paper presented at AERA meetings, Washington, D.C.

ALJSL13H., D., 1963. The Psychology of Meaningful Verbal Learning. (New York: (Yrune y Stratton).

AUSUBEL, D., 1968. Educational Psychology: A cognitive View. (Holt, Rinchart y Winston: New York).

AUSLIBEL, D., NOVAK, D. y HANESIAN, H., 1978. Educational Psychology: A Cognitive View (secondedition). (Holt, Rinehart y Winston: New York).
Deberá, además, profundizarse en investigaciones didácticas futuras; entre otras facetas; en análisis longitudinales de la detección del tipo de rciaciones entre cstas estrategias de aprendizaje significativo y las actitudes de los alumnos en relación a determinados tópicos; en el desarrollo de currículo que suponga un avance en la integración de distintas disciplinas de las ciencias en una estructura conceptual integrada y coherente; en la elaboración de estrategias para modificar aquelias estructuras de conocimiento que contienen errores conceptuales y que influyen negativamente en el aprendizaje posterior; y, finalmente, en la medición de la eficacia de la instrucción mediante mapas conceptuales en la resolución de problemas.

Estamos de acuerdo con Novak cuando señala, refirićndose al Project 2061, Science for All Americans de la American Association for the Advancement of Science (1989): «... concept maps are a step in the right direction foward developing a scientifically literate citizenry by the year 2061».

\section{AGRADECIMIENTOS}

- Al profesor J,D. Novak, por sus enseñanzas y ejemplo. - Al Departamento de Educación de la Universidad de Cornell, por la grata acogida dispensada.

- Al Comité Conjunto Hispano-Americano, por su ayuda económica.

- A M Carmen Manrique Escribano por el mecanografiado del texto.
AUSUBEL, D. NOVAK, J. y HANISIAN, H., 1987. Psicología Educativa. Un punto de Vista Cognoscitivo. (Trillas: Mexico).

BAR-LAVIE, B., 1987. Enhancing Meaningful Learning in an Environmental Education Program: A Case Study of a Class Empowered Through the Use of Novak's and Gowin's Principles of Learning How to Learn, Concept Mapping, Interviewing and Educating. Unpublished $\mathrm{Ph}$. D. thesis. (Cornell University, Department of Education: Ithaca, NY).

BODGEN, CH., 1977. The Use of Concept Mapping as a Possible Strategy for Instructional Design and Evaluation in 
College Genetics. Unpublished Master's thesis. (ComelI University, Department of Education: Ithaca, NY).

BRODY, M. y KOCH, H., 1990. An Assessment of 4th, 8th y 11 th Grade Students' Knowledge Related to Marine Science and Natural Resource Issues, Journal of Environmental Education, Vol. 21 (2), pp. 16-26.

BRODY, M., CHIPMAN, E. y SCOTT, M., 1989. Student Knowledge of Scientific and Natural Resource Concepts Concerning Acidic Deposition, Journal of Environmental Education, Vol. 20 (2), pp. 32-42.

BRUMSTED, CH., 1990. The Degree of Student Engagement in Meaningful Learning Using Cooperative Learning and Student-Selected Subject Matter in Environmental Studies. Unpublished M.S. thesis. (Comell University, Department of Education: Ithaca, NY).

BUCHWEITZ, B., 1981. An Epistemological Analysis of $\mathrm{Cu}$ rriculum and an Assessment of Concept Learning in the Physics Laboratory. Unpublished Ph. D. thesis. (Cornell University, Department of Education: Ithaca, NY).

CARDEMONE, P., 1975. Concept Mapping: A Technique of Analyzing a Discipline and its Use in the curriculum and Instruction in a Portion of a College Level Mathematics Skills Course. Unpublished Master's thesis. (Cornell University, Department of Education: Ithaca, NY).

EDWARDS, J. y FRASER, K., 1983. Concept Maps as Reflectors of Conceptual Understanding, Research in Science Education, Vol. 13, pp. 19-26.

ERSLER, C., 1987. Children's Concepts about Marshes and Ecology before and after Instruction. Unpublished M.S. thesis (Cornell University, Department of Education: Ithaca, NY).

FRASER, K. y EDWARDS, J., 1985. The Effects of Training in Concept Mapping on Student Achievement in Traditional Classroom Tests, Research in Science Education, Vol. 15, pp. $158-165$.

FUATAI, K., 1986. Use of Vee Maps and Concept Maps in the Learning of Form Five Mathematics in Samoa College Western Sumoa. Unpublished Master's thesis. (Comell University, Department of Education: Ithaca, NY).

GONZÁLEZ, F.M., 1991. Los mapas conceptuales de Novak: Una técnica instruccional para la mejora de los procesos de enseñanza/aprendizaje de las ciencias. Príncipe de Viana (suplemento de ciencias).

GOWIN, D.B., 1981, Educating. (Comell University Press: Ithaca, NY).

GURLEY, L., 1982. Use of Gowin's Vee and Concept Mapping Strategies to Teach Responsibility for Learning in $\mathrm{Hi}_{3}$ h School Biological Sciences. Unpublished Ph. D. thesis. (Cornelf University, Department of Education: Ithaca, NY).

HEINZE-FRY, J. y NOVAK, J.D., 1990. Concept Mapping Brings Long-Term Movement toward Meaningful Learning, Science Education, Vol.74 (4), pp. 461-472.

HELM, H. y NOVAK, I., 1983. Proceedings of the International Seminar Misconceptions in Science and Mathematic.s. Department of Education. (Cornell University Press: Ithaca, NY).

KAHLE, J.B., 1984. An Investigation of Instructional Strate- gies which Enhance Biology Meaningfull Leaming. Final Report to the National Science Foundation. (Department of Education, Purdue University, Lafayette, IN).

KINNEAR, J., GLEESON, D. y COMERFORD, C., 1985. Use of Concept Maps in Assessing the Value of a ComputerBased Activity in Biology, Research in Science Education, Vol. 15, pp. 103-111.

MELBY-ROBB, S.J., 1982. An Exploration of the Uses of Concept Mapping With Science Students Labeled Low Achievers. Unpublished M.S. thesis. (Comell University, Department of Education: Ithaca, NY).

MINEMIER, L., 1983. Concept Mapping: An Educational Tool and its Use in a College Level Mathematics Skills Course, Unipublished Master's thesis. (Cornell University, Department of Education: Ithaca, NY).

MOREIRA, M.A., 1988. Mapas Conceptuales en la Finseñanza de la Física, Contactos, Vol. 3 (2), pp. 38-57.

MOREIRA, M.A. y MASINI, F.F., 1982. Aprendizagem Significativa; a Teoria de Aprendizagem de David Ausubel. (Moraes: Sao Paulo).

MOREIRA, M.A. y NOVAK, J.D., 1988. Investigación en Enseñanza de las Ciencias en la Universidad de Cornell: Esquemas Teóricos, Cuestiones Centrales y Abordes Metodológicos, Enseñanza de las Ciencias, Vol. 6(1), pp. 3-18.

NOVAK, J.D., 1982. Teoría y Práctica de lo Educación. (Alianza Universidad: Madrid).

NOVAK, J.D., 1983. Concept-Based I earning, en Kenneth E. Boulding y Lawrence Senesh (eds.), The Optimun Utilization of Knowledge. (Westview Press: Boulder, Colorado), pp. 100-113.

NOVAK, J.D., 1985. Metalearning and Metaknowledge Strategies to Help Students Leam How to Learn, in Leo West y Leon Pines (eds.), Cognitive Structure and Conceptual Change. (In the Educational Psychology Series). (Academic Press: Oriando, Florida), pp. 189-209.

NOVAK, J.D., I988. Constructivismo Humano: Un Consenso Emergente, Enseñanza de las Ciencias, Vol.6(3), pp. $213-$ 223.

NOVAK, J.D., 1991. Clarify with Concepts Maps. A tool for students and teachers alike, The Science Teacher, Vol. 58 (7), pp. 45-49.

NOVAK, J.D. y GOWIN, D.B., 1988. Aprendiendo a Aprender. (Martínez Roca: Barcelona).

NOVAK, J.D., GOWIN, D.B. y IOHANSEN, G., 1983. The Use of Concept Mapping and Knowledge Vee Mapping With Junior High School Science Students, Science Education, Vol. $67(5)$, pp. 625-645

NOVAK, J.D. y staff, 1980. Handbook for the Learning How to Learn Program. (Cornell University, Department of Education: Ithaca, NY).

POSNER, G. y STRIKE, K., 1982. Epistemological Assumptions of College Students: An Initial Report. Paper presented to the $13 \mathrm{th}$. Annual Convocation of the Northeastern Educational Research Association, Ellenville, NY.

ROBERTSON, M., 1984. Use of Videotape-Stimulated Recall 
Interviews to Study the Thoughts and Feelings in an Introductory Biology Laboratory Course. Unpublished M.S. thesis. (Comell University: Ithaca, NY).

ROBERTSON-TAYLOR, M., 1985. Changing the Meaning of Experience: Fmpowering Learners throught the Use of Concept Maps, Vee Diagrams and Principles of Educating in a Biology Lab Course. Unpublished Ph. D. thesis. (Cornell University: Ithaca, NY).

STEWAR'I', J., VAN KIRK, J, y ROWEL,L, R, 1979. Concept Maps: A Tool for Use in Biology Teaching, The American Biology Teacher, Vol. 41 (3), pp. 171-175

STRIKE, K., 1987. Toward a Coherent Constructivism. Proceedings of the Second International Seminar on Misconceptions and Educational Strategies in Science and Mathe. matics. Department of Education. (Comcl1 L'niversify Press: Ithaca, NY).
SYMINGTON, D. y NOVAK, J.D., 1982. Teaching Children How to Learn, The Educational Magazine, Vol. 39 (5), pp. 13-16.

VOLMINK, J., 1983. Meaning in Mathematics: On Integrating Thinking. Feeling and Acting in a First-Year Calculus Course. Unpublished Master's thesis. (Corneli University, Department of Education: Ithaca, NY).

WATERMAN, M., 1982. College Biology Students' Beliefabout Scientific Knowledge: Foundation for Study of Epistemological Commitments in Conceptual Change. Lnpublished doctoral dissertation. (Comell University: Ithaca, NY).

YAAKOBI, DUBA, 1990. C-Map for the Learning Centers. Unpublished Report. (Cornell University Department of Education: Ithaca, NY). 